\title{
Correction to: High-Throughput MicroRNA and mRNA Sequencing Reveals that MicroRNAs may be Involved in Peroxidase-Mediated Cold Tolerance in Potato
}

\author{
Chongchong Yan ${ }^{1}$. Qianqian Wang ${ }^{1} \cdot$ Nan Zhang $^{2} \cdot$ Yuying Fu ${ }^{1}$. Gang Wu ${ }^{1}$. Xueqiang Ren ${ }^{1}$ - Bingjie Xue ${ }^{3} \cdot$ Xiyu Liu $^{4}$. \\ Zhihong $\mathrm{Xu}^{5} \cdot$ Huajun Liao ${ }^{1}$
}

Published online: 23 December 2021

(c) Springer Science+Business Media, LLC, part of Springer Nature 2021

\section{Correction to: Plant Molecular Biology Reporter (2021) 39:577-594 https://doi.org/10.1007/s11105-020-01272-5}

The original version of this article unfortunately contained a mistake. The sequence of the author names was incorrect. All authors agree to make "Huajun Liao" as the corresponding author and "Chongchong Yan" as the first author.

From the previous author order of "Huajun Liao, Qianqian Wang, Nan Zhang, Yuying Fu, Gang Wu, Xueqiang Ren, Bingjie Xue, Xiyu Liu, Zhihong Xu, Chongchong Yan" to "Chongchong Yan, Qianqian Wang, Nan Zhang, Yuying Fu, Gang Wu, Xueqiang Ren, Bingjie Xue, Xiyu Liu, Zhihong $\mathrm{Xu}$, Huajun Liao". And corresponding authorship is now given from "Chongchong Yan" to "Huajun Liao".

Publisher's Note Springer Nature remains neutral with regard to jurisdictional claims in published maps and institutional affiliations.

The original article can be found online at https://doi.org/10.1007/ s11105-020-01272-5.

Huajun Liao

594159401@qq.com

Chongchong Yan

527927758@qq.com

1 Anhui Academy of Agricultural Sciences, Hefei, Anhui, China

2 Anhui Vocational College of City Management, Hefei, Anhui, China

3 Funan County Agricultural Technology Promotion Center, Funan, Anhui, China

4 Linquan County Agricultural Technology Promotion Center, Linquan, Anhui, China

5 Jieshou County Agricultural Technology Promotion Center, Jieshou, Anhui, China 